\title{
Quelle relation entre l'écrit académique et l'écrit professionnel ? Une étude de cas dans le domaine du travail social
}

\section{Theresa Lillis et Lucy Rai}

Traducteur : Frédérique Braem et Dominique Lahanier-Reuter

\section{OpenEdition \\ Journals}

Édition électronique

URL : http://journals.openedition.org/pratiques/1929

DOl : $10.4000 /$ pratiques. 1929

ISSN : 2425-2042

Éditeur

Centre de recherche sur les médiations (CREM)

\section{Édition imprimée}

Date de publication : 15 juin 2012

Pagination : $51-70$

\section{Référence électronique}

Theresa Lillis et Lucy Rai, « Quelle relation entre l'écrit académique et l'écrit professionnel ? Une étude de cas dans le domaine du travail social », Pratiques [En ligne], 153-154 | 2012, mis en ligne le 16 juin 2014, consulté le 01 mai 2019. URL : http://journals.openedition.org/pratiques/1929 ; DOI : 10.4000/ pratiques. 1929 


\section{Quelle relation entre l'écrit académique et l'écrit professionnel ? Une étude de cas dans le domaine du travail social}

\section{Theresa Lillis, Lucy Rai}

The Open University, Royaume-Uni

Texte traduit par Frédérique Braem et

Dominique Lahanier-Reuter

Lille 3, Théodile-CIREL EA 4354

\section{Introduction}

L'enseignement supérieur dispensé au Royaume-Uni s'est considérablement élargi au cours des vingt dernières années, tant au niveau du nombre et de l'éventail des formations proposées qu'en ce qui concerne la taille et la diversité de la population étudiante. La proportion des jeunes de 18-20 ans dans la population globale est passée de $15 \%$ en 1980 à $30 \%$ dans les années 1990 et leur nombre continue d'augmenter, de 270.000 étudiants de premier cycle en 2000 à 360.000 en 2010 (Coleman \& Berkradnia, 2011). En même temps, cette massification s'accompagne d'une croissance importante du taux d'étudiants «non-traditionnels », c'est-à-dire d'étudiants issus de groupes sociaux historiquement exclus de l'éducation supérieure comme la classe ouvrière, issus de groupes ethniques minoritaires et d'étudiants commençant leurs études à plus de 21 ans. Dans ce contexte de changements, on assiste également à une augmentation du nombre de cours professionnalisants au sein de l'enseignement supérieur - et du nombre d'étudiants inscrits à ces cours - en particulier dans les domaines de la santé comme les soins infirmiers et obstétriques et le travail social qui est l'objet de cet article. Cette augmentation du nombre de cours à orientation professionnelle est due en grande partie à la hausse du niveau de qualification universitaire requis, auparavant un diploma, à présent un degree 
level $^{(1)}$, entraînant le transfert de certains cours des centres de formation continue aux universités ${ }^{(2)}$.

En travail social, les cours dispensés à l'université, tout comme d'autres cours professionnalisants, doivent satisfaire à deux types d'exigences : celles des pratiques et valeurs du monde universitaire et celles des pratiques professionnelles et des valeurs du monde du travail. Au carrefour de ces deux domaines, se rencontrent les exigences en matière d'écriture - spécifiques à chacun d'entre eux - et c'est ce que nous nous proposons d'explorer ci-dessous.

En effet, et c'est un point à relever, les productions écrites des étudiants au Royaume-Uni occupent une place particulièrement importante puisqu'elles continuent d'être la modalité d'évaluation la plus utilisée dans les universités et sont, de fait, décisives pour l'obtention d'un degree. L'écriture occupe donc une place centrale au sein de l'université de manière générale (et une position particulièrement complexe en ce qui concerne les formations à orientation professionnelle) et a généré ces dernières années d'importants débats publics et scientifiques.

Le point de vue « déficitaire », c'est-à-dire l'idée que «le niveau baisse » et que «les étudiants ne savent tout simplement plus écrire», est au cœur des représentations des médias et des institutions académiques. On assiste régulièrement à de véritables tollés dans les médias au sujet des faiblesses scripturales des élèves au sortir du système scolaire («They can't read, can't write, keep time or be tidy » ${ }^{(3)}$, The Guardian, 10 mars 2010) et des étudiants ( «niversity students : they can't write, spell or present an argument ${ }^{(4)}$, The Independent, 24 mai 2006), tollés qui s'insèrent dans les affirmations péremptoires concernant le niveau peu élevé en lecture/écriture en général (le journal The Evening Standard, basé à Londres, mène une campagne en 2011 contre le " fléau de l'analphabétisme ») ${ }^{(5)}$. Cette idée d'un déficit dans les universités est souvent associée aux étudiants «non-traditionnels » qui arrivent depuis quelques années à l'université et ressort également dans les débats consacrés à l'écriture dans la formation en travail social, où les étudiants " non-traditionnels » tendent à devenir la norme. La commission sur le travail social (The Social Work Task Force, $S W T F$ ), établie par le gouvernement britannique, a insisté sur la nécessité d'améliorer le "profil des candidats » aux formations en travail social et a proposé pour y parvenir d'introduire un « test écrit permettant de mesurer la clarté de l'écriture, la cohérence du propos et la capacité à adopter une

(1) Diploma est l'équivalent des Brevets supérieurs en France, deux ans d'étude après le lycée, dans des Instituts attachés à des universités, degree est celui de la licence actuelle, trois ans d'étude universitaires.

(2) Le niveau requis en travail social a été relevé du diploma à celui du degree en 2003 (cf. Department of Health 2002) et en soins infirmiers l'obtention d'un degree sera également requise en 2013 ( $c f$. NMC 2010). Bien sûr, la question de savoir dans quelle mesure cette élévation des prérequis aboutit à des étudiants mieux préparés et plus compétents a été soulevée par des universitaires et des professionnels. $C f$., par exemple, Dillon 2011.

(3) «Ils ne savent ni lire, ni écrire, ne savent pas gérer le temps qui leur est imparti ni présenter un écrit soigné».

(4) «Les étudiants à l'université : ils ne savent ni écrire, ni épeler ni argumenter».

(5) $C f$. http://www.thisislondon.co.uk/standard/article-23956817-get-london-reading-jointhe-standards-campaign-to-tackle-illiteracy.do 
posture réflexive et analytique » (SWTF 2009 : 21), cette proposition est actuellement à l'étude (DCSF/DoH 2010). Cette interprétation axée sur le « déficit » s'accompagne d'une approche pédagogique de l'écrit qui domine dans la plupart des institutions du supérieur en Grande Bretagne. On peut la dénommer, de façon globale, approche par les « capacités techniques ». Elle souligne ce que les étudiants " ne savent pas faire », en s'appuyant sur des notions de sens commun concernant les normes de l'écrit académique : les caractéristiques de surface (l'orthographe et la grammaire), les notions réductrices de structure formelle (« introduction », " conclusion ») et les aspects techniques des conventions de citation/renvoi à l'auteur (pour discussion, voir Lea \& Street 1998 ; Lillis 2001 ; Ivanič 2004).

Contrastant avec cette perspective du déficit, s'est développée ce qu'on appelle l'approche des « littéracies universitaires » (academic literacies). Cette approche - minoritaire et opposée à la précédente - s'est développée à partir des travaux de recherche en littéracies (New Literacy Studies), en linguistique et en sciences de l'éducation qui utilisent des méthodologies ethnographiques et valorisent surtout le point de vue des étudiants-auteurs. Une telle perspective a permis de mettre en relief les limites du discours officiel concernant le langage et la littéracie dans le contexte actuel (voir Lillis \& Scott, 2007). Cette approche a été utilisée aussi bien pour étudier les productions écrites des étudiants dans des formations de type plutôt universitaire (e.g. Lillis, 2001, 2009) que dans celles qui revendiquent explicitement une voie professionnalisante (Baynham, 2000 ; Lillis \& Rai, 2011 ; Rai, 2004, 2006).

La position développée dans cet article trouve ses racines dans les travaux sur les littéracies universitaires en Grande Bretagne. Le cadre théorique des littéracies universitaires présente trois dimensions significatives :

1. Épistémologiquement cette approche souligne le fait que l'écriture est toujours une pratique sociale : elle se situe dans un contexte spécifique, avec des participants (réels et immanents) spécifiques, et selon des conventions socio-historiquement élaborées. Bien que ses origines soient différentes, cette approche a beaucoup en commun avec les travaux de Chartier sur la littéracie (1993) et, concernant les écrits universitaires, avec ceux de Rinck (2004).

2. Méthodologiquement, cette approche utilise l'ethnographie ou des approches ethnographiques (Lillis \& Scott, 2007) pour essayer de comprendre l'écriture des participants selon leurs propres perspectives.

3. Idéologiquement, elle adopte une position critique envers le discours dominant dans les médias et les institutions académiques qui souligne les déficits culturels de la population étudiante, et fournit des outils pour regarder au plus près et interroger ces discours dominants.

Cette perspective des littéracies universitaires a permis de mettre en évidence plusieurs dimensions des pratiques d'écriture des étudiants qui restaient invisibles : 1'impact des relations de pouvoir sur 1'écriture étudiante (Lea \& Street, 1998 ; Jones et al., 1999), la centralité de l'identité dans l'écriture étudiante (Ivanič, 1998 ; Lillis, 2001) et l'écriture comme construction idéologi- 
que de la connaissance (Latour, 1979; Lillis \& Scott, 2007). Notre recherche se penche sur l'écriture en travail social, en s'appuyant sur ces trois dimensions pour étudier les articulations entre les pratiques d'écriture académiques et professionnalisantes d'une part et, d'autre part, les relations que ces pratiques d'écriture entretiennent avec les pratiques d'écriture professionnelles dans les contextes du travail social. Les principales questions que cet article soulève sont : 1) dans quelle mesure l'écriture dans la formation au travail social s'apparente-t-elle ou se distingue-t-elle de l'écriture professionnelle ? 2) dans quelle mesure est-elle une préparation à l'écriture professionnelle ?

\section{La place de l'écriture dans la formation au travail social}

L'écriture est fondamentale dans la formation au travail social à l'université, ainsi que dans toutes les formations universitaires. Comme nous l'avons dit plus haut, elle est le principal instrument d'évaluation des connaissances des étudiants et de leur compréhension d'un champ d'étude particulier. Comme dans les autres formations professionnalisantes, les formations diplômantes en travail social à l'université sont un bel exemple de ces études qui ont une double orientation - à la fois universitaire et professionnelle, liée à un domaine spécifique - qui particularise les programmes ; les modalités pédagogiques et d'évaluation sont empreintes à la fois des spécificités de l'enseignement supérieur et de celles des organismes professionnels.

En ce qui concerne les spécificités de l'enseignement supérieur, l'écriture relève de la « communication », considérée comme l'un des six domaines de compétence clés (les autres sont les technologies de l'information, les compétences numériques, les problem solving - résolution de problèmes - et les aptitudes à travailler en groupe) indiqués par la Quality Assurance Agency (QAA) ${ }^{(6)}$. Ceux qui conçoivent et évaluent les cours dispensés à l'université doivent prendre en compte ces domaines de compétence et chercher à les adapter aux programmes des différents niveaux tout au long du cursus universitaire.

Les formations en travail social dans l'enseignement supérieur sont supervisées simultanément par des organismes veillant à développer les compétences et l'expertise que requiert leur profession. Au moment de la recherche sur laquelle cet article se fonde, le curriculum était régi par les National Occupationnal Standards (NOS), les Codes of Practice et par la déclaration des références en travail social (Quality Assurance Agency's benchmark statement for Social Work) de la Quality Assurance Agency (QAA). Le NOS et les Codes of practice régissent les pratiques professionnelles du travail social ainsi que la formation en travail social et ont été établis par un organisme gouvernemental, le General Social Care Council (GSCC). Le NOS décrit les compétences requises des travailleurs sociaux et les Codes of practice définissent les valeurs et l'éthique qui sous-tendent la pratique professionnelle du travail social et de l'aide so-

(6) http://search.qaa.ac.uk/results.aspx?k=The+Framework+for+Higher+Education+Qualifications+in+England\%2C +Wales+and+Northern+Ireland\%2C+available+). 
ciale. La déclaration de la QAA sert de guide pour l'élaboration du curriculum et des critères universitaires pour l'enseignement du travail social et forme, conjointement aux NOS et Codes of practice, ce qui équivaut à un programme national d'enseignement du travail social en Angleterre et au Pays de Galles. Tous ceux qui dispensent des modules de formation qualifiants doivent planifier leur programme et construire leurs modules en fonction de ces trois documents de référence, afin qu'ils soient approuvés et enregistrés par le GSCC. Celui-ci tient actuellement le registre des travailleurs sociaux qualifiés. Bien qu'il n'y ait qu'une seule référence explicite à l'écriture dans les NOS (les travailleurs sociaux doivent être capables d' " expliquer et de justifier (tant verbalement qu'à l'écrit) les motifs de leurs appréciations et décisions professionnelles » (Training Organisation for Personal Social Services (TOPSS), England, 2004 :57), il est fait référence, au moins 46 fois, à l'importance de « l'archivage » (des décisions, des mesures prises, des justifications) ce qui dénote le caractère central de l'écriture dans la pratique du travail social ${ }^{\text {(7) }}$.

La manière dont les spécifications universitaires et professionnelles (explicites et implicites) sont promulguées dans les différentes formations n'est pas claire et il n'y a que peu d'études centrées sur les productions écrites dans la formation au travail social au Royaume-Uni. Cependant, $d$ 'après les travaux existants (Rai, 2004, 2006) il semble que l'on puisse distinguer trois types d'écrits, mais dont deux seulement sont explicitement évalués dans le cadre de la formation : l'essai ${ }^{(8)}$ et l'écrit réflexif. Le troisième type d'écrit est davantage pratiqué dans le lieu de la formation professionnelle plutôt que dans celui de la formation académique, et il semble que l'enseignement et l'apprentissage de ces écrits auraient précisément pour but de se familiariser avec le métier de travailleur social. En effet, ces écrits ne sont pas évalués en tant qu'exercice d'écriture en travail social mais en tant que preuve d'une pratique de travailleur social, c'est-à-dire en tant que preuve que l'on est un bon travailleur social. Un aperçu de ces trois types d'écrits est donné dans le tableau 1 (page suivante).

Bien qu'il y ait de très nombreux conseils et des critères d'évaluation explicites dans ces formations au travail social en ce qui concerne les deux premiers types d'écrits, on n'en trouve pas autant pour le troisième, qui s'inscrit dans le travail que l'étudiant fournit en stage et qui est supervisé par l'organisme partenaire en particulier. Le type d'écriture produit dans le cadre du travail - par exemple les notes de comportement [case notes], les rapports d'évaluation n'est directement évalué ni par l'université, ni par l'organisme partenaire mais il est pris en compte indirectement dans l'évaluation globale des compétences professionnelles de l'étudiant. Là où écriture et pratique professionnelle se rejoignent effectivement dans la formation académique, c'est dans l'attention

(7) Les compétences et normes régissant la formation au travail social sont actuellement à l'étude.

(8) Nous conservons le terme le plus proche de l'anglais essay plutôt que d'adopter la traduction ordinaire, et limitative, de « dissertation », pour réaffirmer la distance entre ces deux types d'écrits. Pour cette raison, « essai » apparaîtra en italique tout au long de cet article. (Note de la traductrice.) 


\begin{tabular}{|c|c|c|c|}
\hline \multirow[b]{2}{*}{ Types d'écrits } & \multicolumn{2}{|c|}{ Explicitement conçu et évalué par l'université } & \multirow{2}{*}{$\begin{array}{l}\begin{array}{l}\text { Indirectement évalué } \\
\text { par l'université dans } \\
\text { le cadre de l'évalua- } \\
\text { tion de la pratique du } \\
\text { travail social }\end{array} \\
\text { 3. Relatif à la pratique }\end{array}$} \\
\hline & 1. Essai & 2. Ecrit réflexif & \\
\hline \multirow[t]{3}{*}{$\begin{array}{l}\text { Caractéristiques } \\
\text { principales }\end{array}$} & $\begin{array}{l}\text { Présentation d'une } \\
\text { discussion }\end{array}$ & $\begin{array}{l}\text { Présentation d'une } \\
\text { réflexion, avant tout } \\
\text { sur les pratiques mais } \\
\text { engageant également } \\
\text { des valeurs } \\
\text { personnelles et } \\
\text { professionnelles }\end{array}$ & $\begin{array}{l}\text { Tâches d'écriture qui } \\
\text { font partie des la } \\
\text { pratique du travail } \\
\text { social ; Notes de } \\
\text { comportement (Case } \\
\text { notes }(9) \text {; rapports } \\
\text { d'évaluation, mails, } \\
\text { par exemple. }\end{array}$ \\
\hline & $\begin{array}{l}\text { Connaissance des } \\
\text { domaines concernés }\end{array}$ & $\begin{array}{l}\text { Connaissance des } \\
\text { domaines concernés }\end{array}$ & \\
\hline & $\begin{array}{l}\text { Utilisation d'éléments } \\
\text { de contenus de cours, } \\
\text { de recherches, des } \\
\text { politiques publiques et } \\
\text { des budgets de la } \\
\text { pratique } \\
\text { professionnelle }\end{array}$ & $\begin{array}{l}\text { Utilisation des } \\
\text { contenus de cours afin } \\
\text { de montrer comment la } \\
\text { réflexion personnelle a } \\
\text { pris appui sur/a tenu } \\
\text { compte des recherches } \\
\text { existantes, des théories } \\
\text { et des mesures } \\
\text { politiques }\end{array}$ & \\
\hline $\begin{array}{l}\text { Évalué en tant } \\
\text { que }\end{array}$ & $\begin{array}{l}\text { production écrite qui } \\
\text { reflète les principales } \\
\text { caractéristiques ci- } \\
\text { dessus et qui est } \\
\text { évaluée par le tuteur } \\
\text { universitaire. }\end{array}$ & $\begin{array}{l}\text { production écrite qui } \\
\text { reflète les principales } \\
\text { caractéristiques ci- } \\
\text { dessus et qui est } \\
\text { évaluée par le tuteur } \\
\text { universitaire. }\end{array}$ & $\begin{array}{l}\text { production écrite qui } \\
\text { atteste d'une pratique } \\
\text { réussie et qui est } \\
\text { évaluée par l'institution } \\
\text { partenaire et } \\
\text { l'institution } \\
\text { d'enseignement } \\
\text { supérieur }\end{array}$ \\
\hline
\end{tabular}

Tableau 1 : Activités d'écriture en travail social à l'université.

très forte accordée à l'écriture réflexive, qui s'inscrit dans la tradition du «praticien réflexif », considéré comme essentiel au développement de praticiens très motivés et critiques. Néanmoins, si la réflexion en tant que pratique personnelle et professionnelle est considérée comme fondamentale, il y a un flou considérable quant à ce que signifie énoncer/produire/représenter une réflexion à l'université (Rai, 2004).

(9) Case notes signifie littéralement : notes sur / pour un cas. (Note de la traductrice.) 


\section{La place de l'écriture dans la pratique professionnelle du travail social}

L'écriture est fondamentale dans la pratique professionnelle : les travailleurs sociaux écrivent dans le cadre de l'évaluation des besoins des bénéficiaires et pour solliciter certains services particuliers afin d'y répondre.

Cependant, il existe d'importantes préoccupations quant à l'écriture dans la pratique des travailleurs sociaux, les deux principales étant : 1) la quantité d'écrits qu'ils sont amenés à rédiger ; 2) la qualité des écrits qu'ils produisent. Concernant le premier point, cette citation du secrétaire général du syndicat illustre bien ces préoccupations liées au temps consacré à l'écriture :

C'est évidemment une erreur que les assistants sociaux passent seulement $20 \%$ du temps avec les familles et les enfants qui ont besoin de leur soutien, et passent le reste du temps à écrire [administration/paperasse] assis devant l'ordinateur. Les assistants sociaux nous disent qu'ils veulent voir inversés ces pourcentages, et ainsi passer $80 \%$ de leur temps avec les bénéficiaires. (Dave Prentis, General Secretary of UNISON, 03/08/09) ${ }^{(10)}$.

Cependant, comme nous le disions précédemment, l'écriture et les compétences linguistiques de certains candidats acceptés dans la formation au travail social dont les lacunes se répercuteront dans leur pratique professionnelle, posent problème :

Nombre d'excellents candidats intègrent la formation et obtiennent leur diplôme en travail social. Cependant, bien qu'il y ait actuellement beaucoup de postes à pourvoir, il apparaît que certaines formations (sans doute en raison de pressions institutionnelles pour que les modules soient remplis) acceptent des candidats dont le profil ne convient pas au niveau requis ou au travail social. Il est à craindre, en particulier, qu'une minorité d'étudiants acceptés dans ces cours n'ait qu'un faible niveau en lecture, en écriture, ou qu'ils soient en difficulté pour analyser et conceptualiser et que la maturité, la résistance ou l'expérience personnelle permettant de devenir de bons travailleurs sociaux ne leur fasse défaut. (DCSF / DoH, 2010:17)

Bien que de récents rapports réalisés pour le gouvernement soulignent l'importance d'améliorer les pratiques et les systèmes d'archivage/d'enregistrement de traces écrites (SWTF, 2009; DCSF / DoH, 2010 ; Munro, 2011) et bien qu'il y ait eu quelques cas très médiatisés où la «communication » a été pointée comme facteur de problèmes (Laming 2009), pour autant que nous le sachions, aucune étude n'a été réalisée à ce jour au Royaume-Uni visant à faire état des exigences en matière d'écriture et des pratiques d'écriture professionnelles et, de fait, ne permet d'étayer les préoccupations dont font état les récents rapports gouvernementaux.

(10) Le syndicat dont la plupart des assistantes sociales sont membres. http://www.unison.org.uk/news/printablepage. asp? $\mathrm{did}=9183$ 
Notre recherche vise à contribuer à l'élaboration d'une cartographie des prérequis en matière d'écriture dans le travail social et étudie en particulier les liens entre les exigences en matière d'écriture, les pratiques d'écriture professionnelles et celles que requiert l'université.

Ainsi, le projet contribue aux débats sur les types d'écrits qui doivent être mis au programme des formations mais aussi aux débats sur la manière dont l'écriture en travail social pourrait être développée et aidée dans le cadre de la pratique professionnelle.

\section{Le projet Getting it write (GiW)}

Dans le cadre d'une étude plus large portant sur 1'écriture professionnelle dans le travail social, nous avons travaillé, pour le projet $G i W$, avec cinq travailleurs sociaux pendant neuf mois. Il s'agissait d'explorer leurs pratiques professionnelles et de prendre la mesure de la cohérence qu'ils voyaient entre l'écriture en formation et celle requise par leur pratique professionnelle du travail social.

Le projet $G i W$ pose trois questions fondamentales. Cet article se focalise principalement sur la première de ces trois questions :

1. Dans quelle mesure l'écriture dans la formation au travail social s'apparente-t-elle ou se distingue-t-elle de l'écriture professionnelle?

2. Dans quelle mesure sert-elle de préparation à l'écriture professionnelle ?

3. Quelles sont les difficultés d'écriture dans le travail quotidien des assistants sociaux?

Afin de répondre à ces questions, nous avons construit ce que nous appelons une « équipe d'ethnographie des textes » (pour une discussion, $c f$. Lillis, 2008, Lillis \& Rai, 2011) avec l'intention de construire une représentation la plus complète possible de l'écriture dans la pratique professionnelle. L'équipe d'investigation est composée de deux chercheurs principaux de 1'Open University, 1'un dans le champ du travail social et l'autre dans celui des academic literacies; les cinq co-chercheurs sont des assistants sociaux [ 2 hommes, 3 femmes] diplômés ces deux dernières années. Tous les co-chercheurs font partie de la protection de l'enfance et travaillent dans cinq organismes différents. Les données collectées comportent les journaux tenus par les travailleurs sociaux où ils tiennent la liste des types d'écrits rédigés, des recueils de productions écrites dans le cadre de leur travail, des entretiens individuels, des discussions de groupe - téléphoniques et en face à face ( $c f$. figure 3 ). L'implication des cinq co-chercheurs a été essentielle à la réussite du projet pour plusieurs raisons, la plus évidente étant qu'ils ont rendu possible l'accès aux textes qu'ils avaient produits et aux pratiques au sein desquelles ces textes prenaient place mais aussi pour le recueil de leur avis critique sur les questions concernant l'écriture au travail. Pendant toute la durée du projet, un temps considérable a été consacré à l'obtention des permissions nécessaires de la part des autorités compétentes afin d'accéder 
aux textes et aux pratiques et pour clarifier les règles de confidentialité qui régissent l'utilisation de ces données hautement confidentielles.

En partant du principe que les principaux types d'écrits présents dans le cursus universitaire nous sont connus, y compris les défis qu'ils peuvent représenter pour les étudiants (voir les travaux de Rai, discutés ci-dessus), un des principaux objectifs de l'étude $G i W$ était d'explorer la nature et les exigences en matière d'écriture dans la pratique du travail social, d'examiner les similitudes et les différences entre elles et d'explorer la pertinence, selon les travailleurs sociaux, des premières par rapport aux secondes. L'objectif était donc d'étudier « l'écriture universitaire » (les tâches d'écriture explicitement conçues et évaluées par l'université) du point de vue du travail social quotidien.

\section{Figure 1 : Résumé des données collectées}

1. 20 entrées des journaux de bord tenus par les co-chercheurs où sont indiqués la portée, le type et la quantité d'écrits rédigés dans leur quotidien professionnel;

2. 200 textes rendus anonymes rédigés dans le cadre de la pratique ;

3. quatre discussions de groupe réunissant chercheurs et co-chercheurs sous la forme de conférences téléphoniques (transcrites) durant la période de collecte de journaux et de textes ;

4. des entretiens individuels avec les co-chercheurs au sujet de leurs pratiques d'écriture et de leurs expériences ;

5. trois jours d'ateliers en face à face à discuter et catégoriser les données collectées.

\section{Les résultats du projet concernant l'écriture professionnelle}

\section{L'écriture est au cœur du quotidien du travail social}

Les journaux tenus sur 20 jours de pratique de travail social montrent que l'écriture prend une place centrale dans l'emploi du temps professionnel. Et en effet, le temps passé à écrire et l'incertitude quant au bénéfice que cela représente pour les usagers des services sociaux les inquiètent :

La plupart du temps nos rapports n'ont rien à voir avec les intérêts de nos usagers. C'est le travail social en face à face que les clients méritent. (TS 2)

Les journaux des cinq travailleurs sociaux montrent que si la quantité d'écrits varie de jour en jour, l'activité scripturale est régulière tout au long de la journée. Le Tableau 2 (page suivante) montre le rythme de l'activité scripturale de chacun des cinq travailleurs sociaux au long de la journée. Les nombres de chaque colonne font référence aux différentes situations spécifiques d'écriture à différents temps de la journée, par exemple écrire un mail = 1 , écrire un rapport ou une partie de rapport $=1$. 


\begin{tabular}{|c|c|c|c|c|c|}
\hline \multirow{2}{*}{$\begin{array}{l}\text { Moment de } \\
\text { la journée }\end{array}$} & TS1 & TS2 & TS3 & TS4 & TS5 \\
\hline & \multicolumn{5}{|c|}{ Nombre d'occurrences individuelles d'écriture } \\
\hline $7 \mathrm{~h} 00$ & & & 1 & & \\
\hline $8 \mathrm{~h} 00$ & & & & 4 & 1 \\
\hline $9 \mathrm{~h} 00$ & 10 & 4 & 1 & 1 & 3 \\
\hline $10 \mathrm{~h} 00$ & 7 & 1 & 3 & 1 & 5 \\
\hline $11 \mathrm{~h} 00$ & 6 & 1 & 6 & 1 & 2 \\
\hline $12 \mathrm{~h} 00$ & 7 & 1 & 7 & 1 & 2 \\
\hline $13 \mathrm{~h} 00$ & 4 & 2 & 5 & 2 & 3 \\
\hline $14 \mathrm{~h} 00$ & 5 & 1 & 1 & 1 & 6 \\
\hline $15 \mathrm{~h} 00$ & 5 & 3 & 2 & 4 & 8 \\
\hline $16 \mathrm{~h} 00$ & 2 & & 3 & & 3 \\
\hline $17 \mathrm{~h} 00$ & 4 & & 1 & 1 & 2 \\
\hline $18 \mathrm{~h} 00$ & & & 1 & & \\
\hline $19 \mathrm{~h} 00$ & & & 2 & & \\
\hline \multicolumn{6}{|l|}{$20 \mathrm{~h} 00$} \\
\hline $21 \mathrm{~h} 00$ & & 1 & & & \\
\hline $22 \mathrm{~h} 00$ & & 2 & & & \\
\hline $23 \mathrm{~h} 00$ & & & & 5 & \\
\hline
\end{tabular}

Tableau 2 : moment de la journée où sont produits les écrits

Remarque : TS 2 a travaillé dans une structure d'accueil résidentiel et selon un horaire irrégulier. Certains de ses écrits ont rédigés durant le temps " calme » tard le soir.

Le Tableau 2 montre également que certains écrits sont rédigés en dehors des horaires contractuels, indiqués en gris. Certains de ces écrits peuvent engendrer beaucoup de temps supplémentaire. Par exemple, les entrées pour une seule journée de TS 4 montrent qu'elle a consacré près de deux heures à des tâches d'écriture après $21 \mathrm{~h} 30$. Dans ce cas, elle a écrit quatre types de textes différents concernant un seul utilisateur du service. Les notes de TS 4 dans son journal donnent une idée de la complexité de la tâche d'écriture : elle indique le nombre de points de vue qu'elle doit prendre en compte en rédigeant ces textes de préparation de dossier (l'utilisateur, la mère, une de ses sœurs, un soignant, un tuteur, la mère biologique, des cadres supérieurs) tout autant que certaines difficultés bureaucratiques qu'elle rencontre pour repérer les bons formulaires. Le défi que peut représenter l'« orchestration » de toutes ces voix (d'après Bakhtine, 1984) fait partie de la recherche et des analyses que nous menons.

Le «U » dans 1'extrait du Tableau 3 (page suivante) fait référence à des temps « ininterrompus », comparativement au «I » des temps « interrompus », un point sur lequel nous allons nous pencher. 


\begin{tabular}{|c|c|c|c|c|}
\hline $\begin{array}{l}\text { Durée } \\
\text { approximative }\end{array}$ & $\begin{array}{l}\text { Lieu } \\
\text { (bureau, } \\
\text { maison, } \\
\text { voiture, } \\
\text { etc.) }\end{array}$ & $\begin{array}{l}\text { Média } \\
\text { (notes } \\
\text { manuscrites } \\
\text { dans un } \\
\text { carnet, } \\
\text { courriel, } \\
\text { système } \\
\text { informatique, } \\
\text { etc.) }\end{array}$ & $\begin{array}{l}\text { Type de textes } \\
\text { (note de } \\
\text { comportement, } \\
\text { courriel, procès } \\
\text { verbal de } \\
\text { réunion, etc.) }\end{array}$ & $\begin{array}{l}\text { Notes sur le texte } \\
\text { (qui était en cause, texte au } \\
\text { stade de brouillon...) }\end{array}$ \\
\hline $\begin{array}{l}21 \mathrm{~h} 38 \\
\text { approx. } \\
10-15 \min (\mathrm{U})\end{array}$ & Maison & $\begin{array}{l}\text { Système } \\
\text { informatique }\end{array}$ & $\begin{array}{l}\text { Notes de } \\
\text { comportement } \\
\text { (Case notes) }\end{array}$ & $\begin{array}{l}\text { Jeune } \\
\text { et soignant }\end{array}$ \\
\hline $\begin{array}{l}22 \mathrm{~h} 07 \\
\text { approx. } \\
30 \text { min. (U) }\end{array}$ & Maison & $\begin{array}{l}\text { Système } \\
\text { informatique }\end{array}$ & Référé & $\begin{array}{l}\text { Mère biologique, assistant en } \\
\text { soutien familial, responsable } \\
\text { et assistant social. Il m'a } \\
\text { fallu des jours pour obtenir le } \\
\text { formulaire d'orientation pour } \\
\text { que la procédure puisse être } \\
\text { faite. J'ai envoyé de } \\
\text { nombreux courriels et passé } \\
3 \text { appels téléphoniques avant } \\
\text { de repérer le bon formulaire. } \\
\text { J'avais en fait complété un } \\
\text { mauvais formulaire et j'ai dû } \\
\text { recommencer. }\end{array}$ \\
\hline $\begin{array}{l}22 \mathrm{~h} 49 \\
\text { approx. } \\
1 \text { heure (U) }\end{array}$ & Maison & $\begin{array}{l}\text { Système } \\
\text { informatique }\end{array}$ & $\begin{array}{l}\text { Placement avec } \\
\text { accord parental }\end{array}$ & $\begin{array}{l}\text { Discussion avec le juge au } \\
\text { tribunal et le tuteur. } \\
\text { Discussion avec la mère } \\
\text { biologique et sa sœur en } \\
\text { Irlande. Discussions avec } \\
\text { mon directeur et les cadres } \\
\text { supérieurs. }\end{array}$ \\
\hline $\begin{array}{l}23 \mathrm{~h} 51 \\
\operatorname{approx} . \\
3 \min (\mathrm{U})\end{array}$ & Maison & $\begin{array}{l}\text { Système } \\
\text { informatique }\end{array}$ & $\begin{array}{l}\text { Conversation } \\
\text { téléphonique } \\
\text { Note de } \\
\text { comportement } \\
\text { (Case note) }\end{array}$ & $\begin{array}{l}\text { Discussion avec les proches } \\
\text { de la mère biologique. } \\
\text { Recherche d'un accord }\end{array}$ \\
\hline
\end{tabular}

Tableau 3 : extrait du journal de TS 4 illustrant les activités d'écriture en dehors du temps de travail.

\section{On écrit souvent d'une façon fragmentée}

En raison de la pression actuelle, on écrit en marge du « travail », de manière fragmentée. Les écrits interrompus (c'est-à-dire lorsque le travailleur social commence à rédiger mais doit s'interrompre pour s'atteler à d'autres tâches puis revient à un moment différent à son écrit) sont assez fréquents. Plus de $40 \%$ des écrits recensés dans les journaux rédigés sur le temps de travail sont des écrits interrompus. Réussir à rédiger de cette façon représente une forme de 
défi, en particulier pour les types d'écrits les plus complexes, tels ceux décrits ci-dessus ( $c f$. Tableau 3) qui impliquent d'intégrer les points de vue de beaucoup de personnes. Nous avons déjà mentionné que certains écrits étaient rédigés en marge du temps de travail, souvent chez soi, et une des raisons en est que cela offre aux travailleurs sociaux le temps et le lieu pour travailler sur des écrits plus complexes.

Les défis auxquels les travailleurs sociaux sont confrontés par rapport à la gestion des demandes d'écrits sur leur temps de travail, à côté d'autres tâches, est illustré par l'extrait d'un des journaux ci-dessous :

Heure : 19h39. Lieu : au bureau

Je suis retourné au bureau suite à ma visite à domicile et $\mathrm{j}$ 'ai tapé les commentaires. Si nous n'enregistrons pas les visites à domicile dans la section "visites » du système, il n'y a aucune preuve que la visite a eu lieu (si elle n'est pas consignée, elle n'a pas eu lieu). Il est rare que j'aie l'opportunité de consigner une visite à domicile juste après l'avoir faite, mais c'était un nouveau cas et mes enregistrements commencent bien pour les cas nouveaux parce que je ne veux pas prendre de retard comme ce qui a tendance à arriver pour les cas dont je m'occupe depuis longtemps. La plupart des visites à domicile que j'effectue se font après le temps scolaire, quand on peut voir les enfants à partir de $16 \mathrm{~h}$. Cela veut dire que les visites à domicile se terminent après la fin de notre journée de travail et, à moins de retourner au bureau, les comptes rendus de ces visites sont souvent négligés.

Les travailleurs sociaux sont impliqués dans beaucoup de tâches d'écriture complexes

Grâce aux journaux, on a identifié au moins 21 types de textes, listés dans le tableau 4 ci dessous. Les textes marqués d'un $\sqrt{ }$ sont ceux qui sont établis selon un modèle particulier qui spécifie quelles sont les informations et les évaluations requises par l'institution et comment celles-ci doivent être structurées.

\begin{tabular}{|l|l|c|}
\hline & Types d'écrits & Modèle \\
\hline 1 & Référés judiciaires / référés & $\sqrt{ }$ \\
\hline 2 & Évaluation de base & $\sqrt{ }$ \\
\hline 3 & Rapport pour la Conférence de protection de l'enfance & $\sqrt{ }$ \\
\hline 4 & Notes manuscrites (sur les visites à domicile) & \\
\hline 5 & Réunion du groupe de base & $\sqrt{ }$ \\
\hline 6 & Information sur un placement & $\sqrt{ }$ \\
\hline 7 & Formulaire financier & $\sqrt{ }$ \\
\hline 8 & Comptes rendus de réunion (entre professionnels) (plan de sécurité) & \\
\hline 9 & Comptes rendus de réunions d'analyse / réunion d'analyse des soignants & \\
\hline 10 & Enregistrement d'observation & \\
\hline
\end{tabular}




\begin{tabular}{|l|l|c|}
\hline 11 & Note de comportement (Case note) & \\
\hline 12 & Courriel & \\
\hline 13 & Plan de supervision & $\sqrt{ }$ \\
\hline 14 & Mise à jour / communications ; actualités... etc. & $\sqrt{ }$ \\
\hline 15 & Lettre / projet de lettre & $\sqrt{ }$ \\
\hline 16 & Convocations pour infractions & $\sqrt{ }$ \\
\hline 17 & Déclaration judiciaire & $\sqrt{ }$ \\
\hline 18 & Message texte & $\sqrt{ }$ \\
\hline 19 & Examen de placement & $\sqrt{ }$ \\
\hline 20 & Demande de transfert & \\
\hline 21 & Placement avec accord parental & \\
\hline
\end{tabular}

Tableau 4 : types de textes consignés dans les journaux ${ }^{(11)}$.

Les types d'écrits varient en complexité, de courts e-mails informatifs à la mise au point de rapports complexes. Les textes les plus courts recueillis sur ces 20 jours sont des courriels d'une seule ligne, le texte le plus long comptait 25 pages. L'inquiétude quant à la valeur que représentent des textes aussi longs et quant aux bénéfices qui en seront effectivement tirés est très ressentie :

Ces rapports sont partagés avec les enfants et les familles avec qui on travaille... ce n'est pas utile pour eux de recevoir des documents de quarante pages qui ont plus à voir avec l'organisation et l'information dont celle-ci a besoin. (TS5)

Bien sûr, la complexité d'une tâche d'écriture (ainsi que sa durée) ne se juge pas nécessairement à la longueur d'un texte spécifique, bien que, dans ce contexte, cette longueur soit souvent un bon indicateur de la complexité et du temps considérable que la tâche implique.

Par ailleurs, ce sont souvent les relations entre les textes qui présentent des difficultés. À cet égard, il est important de noter que plus d'un tiers des textes produits par les travailleurs sociaux sont des « comptes rendus ». Les comptes rendus représentent un aspect fondamental de la pratique du travail social, ils servent à tenir le compte des activités spécifiques des travailleurs sociaux, et justifient les types particuliers d'interventions auprès des usagers du service. Ces textes représentent des défis particuliers pour les travailleurs sociaux parce qu'ils fonctionnent institutionnellement plutôt comme une " chaîne de texte » (Fraenkel, 2001) que comme des textes isolés; à savoir, chaque note de comportement fait partie de l'histoire d'un cas, et les éléments clés - description (d'une personne, d'un problème, d'une situation), évaluation professionnelle, articulation de décisions et liste des actions entreprises, y compris leurs

(11) La classification des types de textes a été pensée avec les co-chercheurs. Des analyses plus poussées seront faites pour établir les relations entre ces différents types de textes. 
justifications - doivent apparaître à intervalles réguliers dans cette chaîne de textes particulière, il n'est pas évident de savoir exactement quand et comment chacun de ces éléments doit y figurer.

Par ailleurs, les notes de comportement font partie d'une chaîne plus large de textes dans laquelle elles sont reconfigurées et sont utilisées dans d'autres documents, tels que des rapports formels. Un exemple tout à fait particulier des difficultés que soulèvent les recontextualisations de ces notes fut évoqué dans l'un des groupes de discussion. Un travailleur social pointait les difficultés qu'il avait rencontrées en tentant de rendre compte fidèlement de l'attitude d'un jeune dans un rapport pour le tribunal. Dans ses notes de comportement, le travailleur social avait consigné textuellement ce que la personne avait dit, y compris les insultes qu'elle avait proférées à son égard et à celui des autres travailleurs sociaux, parce qu'en général, il estime que rapporter les paroles exactes rend mieux compte des événements et des comportements des gens :

Dans nos comptes rendus, nous écrivons ce qui est dit et cela montre combien les mots peuvent vraiment être puissants. Si nous ne saisissons pas ce qui est réellement dit, nous pouvons mésestimer une situation, vous savez, et nous mettre en danger ou mettre d'autres en danger. (TS1)

Mais dans la rédaction d'un rapport officiel pour les tribunaux au sujet de cette personne, son directeur lui a indiqué qu'il n'était pas souhaitable de faire apparaître de tels « jurons ». Dans un rapport de tribunal, la transcription des insultes a été coupée et remplacée par une description :

Durant notre discussion, $\mathrm{X}$ est devenu de plus en plus agité et en colère quant au fait de ne s'impliquer dans aucune de ses sessions. Il est devenu verbalement agressif envers les professionnels, proférant envers eux insultes et menaces. Tous les efforts pour encourager X à s'impliquer sans être violent et menaçant ont échoué. [Extrait du rapport de TS1]

Le travailleur social a estimé que le fait de ne pas rapporter les termes réellement utilisés à l'oral par l'adolescent a eu pour effet de minimiser l'agressivité dont il avait fait preuve et que cela avait orienté la décision du tribunal, de ne prendre aucune mesure envers ce jeune. Dans d'autres cas, ainsi que nous le verrons, les propos exacts sont inclus dans les rapports formels.

\section{Un contenu souvent très difficile émotionnellement mais qui doit être écrit} de manière détachée, rapide, souvent sans le soutien d'un supérieur.

Une des raisons pour lesquelles l'écriture - tant des textes plus courts que des textes plus longs - peut être particulièrement difficile est que le contenu des propos est souvent très difficile émotionnellement bien que le travailleur social ait à rédiger de manière détachée. La plupart des 200 textes collectés sont construits autour de descriptions et d'évaluations de situations très fortement chargées émotionnellement, le bref extrait ci-dessous est issu d'un rapport initial au tribunal : 
J'ai demandé à X pourquoi elle avait dit à un travailleur social que XX avait violé XXX : elle a répondu " XX m'a dit que XXX la battait sur les fesses et lui donnait la fessée ». XX a déclaré qu'elle avait interrogé XXX, lui a demandé pourquoi il l'avait fessée. Elle a dit que c'est alors qu'elle a découvert que XXX avait menti, en réalité. (Extrait de rapport. TS4]

Bien qu'il ne soit pas surprenant de trouver de tels écrits dans la pratique du travail social, il est important de reconnaître les difficultés particulières qu'ils représentent, en particulier, tel que nous l'avons dit précédemment, quand tant d'écrits sont produits de manière fragmentée.

Je trouve ça souvent extrêmement difficile, et il y a une zone particulière de difficultés pour moi qui est dans le travail sur les histoires de vie, puis les lettres de vie, alors quand je dois rendre compte à des enfants de ce qui s'est passé au cours de leur vie, pourquoi certaines décisions ont été prises, pourquoi leurs parents n'étaient pas en mesure de prendre soin d'eux et le faire d'une manière accessible. Extrêmement difficile. Et ça me prend des heures d'écrire, je commence et j'efface et j'écris et je pense juste que je ne sais pas comment dire ça à cet enfant. C'est tellement douloureux. (TS 3)

Et les travailleurs sociaux sentent souvent qu'ils n'ont pas assez de temps à consacrer à l'élaboration et à la rédaction de ces textes :

Une chose avec laquelle j'ai du mal dans mon travail quotidien, c'est que je sens bien que je n'ai ni le temps ni la capacité d'écrire les rapports avec autant de détails que je le souhaiterais. (TS5)

Étant donné le caractère fragmenté de la rédaction, écrire des notes de comportement et d'autres documents complexes est difficile. Les travailleurs sociaux disent qu'il est difficile d'écrire au niveau considéré comme acceptable.

\section{Les résultats du projet quant à la relation entre l'écriture professionnelle et l'écriture en formation}

En se basant sur les propos qui précèdent, il est possible de résumer les principales différences entre l'écriture dans le domaine universitaire et l'écriture dans le domaine professionnel (Tableau 5 page suivante).

Tous les travailleurs sociaux ont fait remarquer que les contraintes d'écriture auxquelles ils seraient confrontés dans leur pratique professionnelle sont l'objet de très peu - ou pas du tout - d'attention dans les programmes universitaires, et qu'elles devaient être apprises au travail :

Ils [les professeurs] ne parlent pas des types de rapports écrits par les assistantes sociales - que ce soit des assistantes sociales de la protection de l'enfance, des service d'adoption, des services de jeunes délinquants - il est presque absent, ce module où l'on vous dit : Ok voilà la journée d'un travailleur social et voilà les types de rapports qu'on attendra de vous et voici ce qu'est le format ou un formulaire E ou une enquête relative à la protection de l'enfance. (TS 3) 


\begin{tabular}{|l|l|}
\hline $\begin{array}{l}\text { Que sait-on de l'écriture dans la } \\
\text { formation ? }\end{array}$ & $\begin{array}{l}\text { Que sait-on de l'écriture dans la pratique } \\
\text { professionnelle ? }\end{array}$ \\
\hline $\begin{array}{l}\text { Une gamme assez restreinte de textes - deux } \\
\text { types d'écrits principaux - les essais et les } \\
\text { écrits « réflexifs » }\end{array}$ & $\begin{array}{l}\text { Une large gamme de textes - 21 types } \\
\text { d'écrits dans cette étude, des courriels brefs } \\
\text { aux documents plus longs et complexes. }\end{array}$ \\
\hline $\begin{array}{l}\text { Les textes tendent à fonctionner en tant que } \\
\text { textes isolés }\end{array}$ & $\begin{array}{l}\text { Les textes tendent à fonctionner dans des } \\
\text { chaînes de textes }\end{array}$ \\
\hline $\begin{array}{l}\text { Les textes sont lus et évalués par un } \\
\text { « expert », le professeur }\end{array}$ & $\begin{array}{l}\text { Les textes sont lus et évalués par nombre } \\
\text { d'« experts »/de gens aux angles de vue } \\
\text { variés (bénéficiaires, directeurs, police, } \\
\text { juges, personnels de santé) }\end{array}$ \\
\hline $\begin{array}{l}\text { Les textes présentent les points de vue d'un } \\
\text { seul auteur }\end{array}$ & $\begin{array}{l}\text { Les textes présentent des voix multiples - le } \\
\text { travail des assistantes sociales est de } \\
\text { coordonner les informations de beaucoup de } \\
\text { sources et de perspectives diverses } \\
\text { (médecins, familles, professeurs...) }\end{array}$ \\
\hline $\begin{array}{l}\text { L'objectif est de montrer qu'on apprend } \\
\text { selon les attentes des professeurs, du } \\
\text { curriculum et de l'idéologie du travail social, } \\
\text { du point de vue de l'imaginaire disciplinaire } \\
\text { académique }\end{array}$ & $\begin{array}{l}\text { L'objectif est de montrer qu'on a bien } \\
\text { pompris l'information et les perspectives de } \\
\text { plusieurs personnes et évènements }\end{array}$ \\
\hline $\begin{array}{l}\text { On sait à l'avance quand on doit avoir fini un } \\
\text { écrit et organiser le temps nécessaire pour y } \\
\text { parvenir }\end{array}$ & $\begin{array}{l}\text { En raison de la pression horaire, on écrit en } \\
\text { marge du « travail », de façon fragmentée }\end{array}$ \\
\hline $\begin{array}{l}\text { 'écrit a une vie courte - c'est fini quand on } \\
\text { a été évalué... }\end{array}$ & \begin{tabular}{l} 
L'écrit a une longue vie... sans fin... \\
\hline Tableau : Rén
\end{tabular} \\
\hline
\end{tabular}

Tableau 5 : Résumé des principales différences entre l'écriture dans le domaine universitaire et le domaine professionnel.

Cependant, plusieurs d'entre eux ont estimé que les types d'écrits effectués dans la formation - les essais et écrits réflexifs - leur avaient appris à être précis et critiques, ce qui est important dans leurs écrits professionnels.

Je crois qu'autant qu'à s'asseoir et à écrire un rapport très précis, alors oui, je crois que l'apprentissage autour de " comment écrire en adoptant une posture critique » et analyser, c'est profitable...

Et

J'avais pris l'habitude de broder vraiment n'importe comment, vous voyez - je vais bien plus franchement dans le vif du sujet, au lieu d'utiliser cinq ou six phrases pour faire une remarque, je le fais bien plus rapidement. (TS 2)

(12) Il y a bien sûr un large éventail de tâches d'écriture dans ces catégories, mais pour l'objet de notre étude, elles sont une entrée utile pour commencer à relever différences et similarités entre les écrits universitaires et professionnels. 
L'une d'entre eux a expliqué combien l'écriture dans la formation universitaire avait pu, de façon implicite, lui apprendre l'importance de la langue et le pouvoir des mots :

Vous commencez par apprendre comment présenter l'information de la manière la plus efficace. Alors vous commencez à comprendre que si vous écrivez de la sorte, vous pouvez probablement influencer les gens qui vous lisent de manière positive ou négative - ça fait impression et ça vous représente, vous apprenez que si vous écrivez d'une façon particulière alors c'est plus simple pour les gens de comprendre - et vous savez, votre public étant juge, il détermine qui est le plus fort ou qui semble proposer l'avis le plus réaliste, le plus raisonnable quant à ce qui a eu lieu ou n'a pas eu lieu. Je pense qu'on oublie parfois ça mais je pense qu'inconsciemment nous l'intériorisons. (TS 5)

La même personne s'est toutefois exprimée sur le fait qu'elle n'avait pas assez de temps pour consacrer autant d'attention à l'utilisation de la langue dans l'élaboration de ces rapports, ce qu'elle regrettait profondément.

Vous savez, j'ai comme l'impression que ma pratique d'écriture, comparativement à la rédaction de mes essais, est assez pauvre aujourd'hui et ce n'est pas du niveau de ce que je voudrais produire. Je pense que c'est une chose contre laquelle je lutte dans ma pratique quotidienne, je ne pense pas avoir le temps qu'il faut, la capacité à écrire n'importe quoi, à rédiger les rapports, la qualité des rapports que je veux écrire. (TS 5)

De la même manière, l'importance qu'attachent le monde universitaire et les travailleurs sociaux aux écrits réflexifs ne trouve que peu de place dans les pratiques d'écriture professionnelles. Un des travailleurs sociaux pointait le contraste qu'il pouvait y avoir entre l'engagement dans une réflexion auquel on est encouragé durant la formation universitaire et les réalités de la pratique du travail social. Quelques propos de travailleurs sociaux qu'il a observés lorsqu'il a commencé à travailler :

Je pense qu'il y avait des travailleurs sociaux fantastiques, mais ils n'avaient pour ainsi dire pas le temps d'écrire, vous voyez, ni le temps de réfléchir à ce qu'ils écrivaient. (TS2)

Même si on regrette que peu d'attention, voire aucune attention, n'ait été accordée dans la formation universitaire à l'enseignement de l'écriture professionnelle, il y a consensus à admettre qu'il est probablement impossible de préparer les futurs travailleurs sociaux aux tâches d'écriture complexes qu'ils vont être amenés à produire au travail.

Tandis qu'on peut enseigner des techniques d'écriture précises (par exemple, comment écrire un rapport), il n'est pas possible d'enseigner par avance ce qu'est la réalité de l'écriture au travail. (TS4)

Ceci suppose que la façon d'apprendre ce qu'est l'écriture professionnelle, en travail social, est de le faire en contexte professionnel, ainsi que le pointe Paré dans son étude sur le travail social au Canada (Paré, 2000 ; 2004). Pour- 
tant, notamment en raison des contraintes horaires, il semble qu'il y ait peu de temps pour enseigner et apprendre au travail. Néanmoins, l' « impact» des difficultés liées à l'ampleur et à la variété des écrits au travail peut être considérable pour les travailleurs sociaux :

Je pense que ça n'a pas été un choc pour moi parce que je consignais et j'écrivais déjà des rapports depuis des années, mais ça peut faire un choc à quelqu'un de nouveau dans le métier, de découvrir combien il faut être impliqué et polyvalent pour monter des dossiers, pour composer les différents types d'écrits auxquels vous êtes confronté. Ça s'est bien passé pour moi, mais ça met quand même en lumière que vous devez consigner beaucoup de choses de bien des façons différentes - dans la manière d'écrire et dans bien des styles différents. Il faut beaucoup de temps pour acquérir ça, vous savez, et faire des progrès chemin faisant. (TS1)

\section{Conclusion}

Il existe des différences majeures entre le monde universitaire et le monde professionnel, en termes de types de textes et de pratiques qu'ils façonnent. Le degré d'adéquation des types d'écrits enseignés à l'université au monde du travail est variable : certains travailleurs sociaux soulignent l'absence de cohérence tandis que d'autres ont éprouvé l'utilité d'une des approches générales, telles que 1'apprentissage des tournures rhétoriques pour façonner un écrit ou le fait d'adopter une posture critique et réflexive dans leur travail, et par conséquent dans leur écriture. Pour le moment, les écrits professionnels ou basés sur la pratique ne sont pas inscrits dans les programmes universitaires, mais apparaissent seulement de façon indirecte dans l'évaluation de la pratique globale. Parallèlement, il y a peu d'espace dans le domaine professionnel ou dans le monde du travail pour enseigner et apprendre comment produire les types d'écrits qui sont au centre de la pratique professionnelle quotidienne. Étant donnée la quantité d'écrits que les travailleurs sociaux sont amenés à rédiger, la complexité de certaines tâches et les conséquences importantes que peuvent engendrer de tels écrits (tant pour les travailleurs sociaux que pour les bénéficiaires de leurs services), il est clairement nécessaire de chercher à savoir où, comment et quand les écrits professionnels peuvent être enseignés. 


\section{Références}

BAKHTINE, M. (1984) : Esthétique de la création verbale, traduit par A. Aucouturier, Paris, NRF Gallimard.

BAYNHAM, M. (2000) : "Academic Writing in New and Emergent Discipline Areas », in : Lea, M.R., Stierer, B. (eds.), Student Writing in Higher Education: New Contexts. Milton Keynes. The Society for Research into Higher Education \& Open University Press : 17-31.

Chartier, R.(1993) : Pratiques de la lecture. Paris . "Petite Bibliothèque Payot $\gg n^{\circ} 167$.

CHILDREN'S WORKFORCE DEVELOPMENT COUNCIL (2010) : Step up to Social work http ://www.cwdcouncil.org.uk/social-work/step-up-to-social-work Accessed 22/06/11.

Coleman, R. \& BerKRADNiA, B (2011) : Higher Education Supply and Demand to 2020 Higher Education Policy Institute. http ://www.hepi.ac.uk/455-1907/Higher-Education-Supply-and-Demand-to-2020.html. Accessed : 11/01/12.

DEPARTMENT FOR CHILDREN, SCHOOLS AND FAMILIES / DEPARTMENT OF HEALTH (DCSF/DoH) (2010) : Building a safe and confident future : Implementing the recommendations of the social work task force. HMSO London.

DILlON, J. (2010) : « Black minority ethnic students' journeys to higher education : realisable or thwarted ambitions ? ». The International Journal of Learning, 17(2) : 219-231.

FRAENKEL, B. (2001) : «Enquêter sur les écrits dans l'organisation », Borzeix, A., Fraenkel, B. (eds.), Language et travail. Communication, cognition, action. Paris. CNRS Editions : 231-261.

IVANIČ, R. (2004) : « Discourses of Writing and Learning to Write ». Language and Education, 18(3) : 220-245.

Jones, C., Turner, J. \& STREeT, B. (eds.) (1999) : Students writing in the university : Cultural and epistemological issues, Amsterdam : John Benjamins.

LAMING, H., (2009): The protection of children in England: A progress report. HMSO, London.

LEA, M. \& STREET, B. (1998) : «Student writing in higher education : an academic literacies approach ». Studies in higher education. 23(2) : 157-172.

LEA, M. \& STIERER, B., (eds.) (2000) : Student writing in higher education: new contexts. Buckingham : Society for Research into Higher Education and The Open University Press.

LILLIS, T. (2001) : Student writing : Access, Regulation, Desire, London : Routledge.

- (2008) : «Ethnography as method, methodology and "deep theorising”: closing the gap between text and context in academic writing research ». Written Communication, 25, $3: 353-388$.

— (2009) : «Étudiants déficitaires ? Le dialogisme de Bakhtine dans 1'ex- 
ploration des pratiques d'écriture des étudiants "non traditionnels" à l'université ». Cahiers Théodile, $10: 117-132$.

LILLIS, T. \& RAI, L. (2011) : «A case study of a research-based collaboration around writing in social work », Across the Disciplines 8 (3), http ://wac.colostate.edu/atd/clil/lillis-rai.cfm.

LILLIS, T. \& SCOTT, M. (2007) : «Defining academic literacies research : issues of epistemology, ideology and strategy ». Journal of Applied Linguistics. 4 (1) : 5-32.

MUNRO, E.,(2011): The Munro review of child protection. Final report : a childcentred system. HMSO, London.

NURSING AND MIDWIFERY COUNCIL (NMC) (2010) : Standards for Pre-registration nursing education. http ://standards.nmc-uk.org/Pages/Welcome.aspx. Accessed : 8/10/11.

PARÉ, A. (2000) : «Writing as a way into social work: Genre sets, genre systems, and distributed cognition ", in Dias, P., Paré, A. (eds.) Transitions ; Writing in academic and workplace settings. Hampton Press, New Jersey.

- (2004) : « Texts and power : Towards a critical theory of language», in Davies, L., Leonard, P. , Social work in a corporate era. Ashgate, Aldershot.

RAI, L. (2004) : «Exploring literacy in social work education ». Social Work Education, 23, 2 : 149-162.

- (2006) : «Owning (up to) reflective writing in social work education». Social Work Education, 25, 8 : 785-797.

RINCK, F. (2004) : «Les difficultés d'étudiants dans la construction d'une problématique », Pratiques, 121/122: 93-110.

SOCIAL WORK TASK FORCE REPORT (SWTF) (2009) : Building a safe and confident future: Final report of the social work task force. HMSO London.

Training ORganisation fOr PERSONAl Social SERVICES (TOPSS) England (2004) : National Occupational Standards (NOS) for social work. TOPSS, Leeds. 